\title{
Magnetic Excitations in Locally Non-Equilibrium Continuum
}

\author{
A.F. KABYCHENKOV* \\ Fryazino Filial of V.A. Kotel'nikov Institute of Radioengineering and Electronics of RAS \\ Vvedensky sq. 1, Fryazino, Moscow region, 141190, Russia \\ (Received March 21, 2012; in final form January 25, 2013)
}

\begin{abstract}
On the basis of locally non-equilibrium thermodynamics the equation for dynamics of the magnetic moment vector is derived. For a magneto disordered continuum the spectrum of locally non-equilibrium fluctuations is determined. It is shown that the spectrum is composed of electromagnetic-spin branches, which contain energy gaps and spin-electromagnetic ones which do not. Unstable modes in diamagnets are found. The dispersion relation and frequency dependence of the damping coefficient are determined for coupled waves of the magnetic vector potential and magnetization. It is shown that there exist frequency regions of transmission and non-transmission. Frequencies of the undamped waves and constant-phase damped waves can be located in these regions.
\end{abstract}

DOI: 10.12693/APhysPolA.123.695

PACS: $41.20 .-\mathrm{q}$

\section{Introduction}

Waves of magnetization in a locally equilibrium dielectric continuum have been considered in a number of papers [1]. In this paper coupled waves of the magnetic vector potential and magnetization in a locally non-equilibrium magneto non-ordered continuum in absence of external fields [2] are investigated. This continuum can be a model for paramagnetic and diamagnetic materials, spin glasses, nuclear spins, a neutron continuum.

Coupled waves of an electromagnetic (EM) field and magnetization are similar to sound waves in the continuum with spins [3]. Interaction of the vector potential (impulse) and magnetic moment vector (spin) fields represents a real model for the field theory.

\section{Equation of motion for the magnetic moment vector in a locally non-equilibrium continuum}

Equation of motion for magnetization can be derived within the frame of non-equilibrium thermodynamics $[4,5]$. According to this theory magnetization obeys the conservation equation (CE):

$$
\boldsymbol{M}_{, t}+\boldsymbol{R}_{j, j}=\boldsymbol{N}
$$

where (.), ${ }_{t} \equiv \partial(.) / \partial t$ and (.),,$j \equiv \partial(.) / \partial x_{j}$ are the derivatives with respect to time and space, $\boldsymbol{R}_{j}=R_{j i} \boldsymbol{e}_{i}, \boldsymbol{e}_{i}$ is the basis vector, $R_{j i}$ is the $j$-component of the flow of $M_{i}$-component (surface force moment density), $\boldsymbol{N}$ is the source of $\boldsymbol{M}$ (volumetric force moment density).

The electromagnetic equations are the CE's with antisymmetric tensors of the flows. On the basis of the equations for dielectric continuum the EM field energy satisfies the $\mathrm{CE} w_{, t}^{\mathrm{f}}+\operatorname{div} \boldsymbol{J}^{\mathrm{f}}=\sigma^{\mathrm{f}}$, where $w^{\mathrm{f}}=(8 \pi)^{-1}\left(\boldsymbol{E}^{2}+\right.$ $\left.\boldsymbol{B}^{2}\right)-\boldsymbol{M B}$ is the energy density, $\boldsymbol{E}$ is the electric field, $\boldsymbol{B}=\boldsymbol{H}+4 \pi \boldsymbol{M}$ is the magnetic induction, $\boldsymbol{H}$ is the magnetic field, $\boldsymbol{J}^{\mathrm{f}}=(c / 4 \pi)[\boldsymbol{E} \boldsymbol{H}]$ is the density of the field

\footnotetext{
*e-mail: akab@mail.cplire.ru
}

energy flow, c is the light velocity, $\sigma^{\mathrm{f}}=-\boldsymbol{P}_{, t} \boldsymbol{E}-\boldsymbol{M}_{, t} \boldsymbol{B}$ is the density of the energy source, $\boldsymbol{P}$ is the electric polarization [4].

The internal energy defined as difference between the total energy and the EM field energy satisfies the CE $u_{, t}+\operatorname{div} \boldsymbol{q}=-\sigma^{\mathrm{f}}$, where $u\left(s, P_{i}, M_{j}\right)$ is the internal energy density, $s$ is the entropy density, $\boldsymbol{q}$ is the internal energy flow.

On the base of CE's for $u$ and $M$ at constant polarization one can write the $\mathrm{CE}$ for entropy as

$$
s_{, t}+J_{j, j}^{\mathrm{s}}=\sigma,
$$

where $\boldsymbol{J}^{\mathrm{s}}$ is the entropy flow. The entropy production is the positive-definite bilinear form

$$
\sigma=\boldsymbol{J}^{n} \boldsymbol{K}^{n} \geq 0
$$

in which the generalized thermodynamic flows and thermodynamic forces conjugate to them are

$$
\begin{aligned}
& \boldsymbol{J}^{1}=\boldsymbol{J}^{\mathrm{s}}, \quad \boldsymbol{K}^{1}=(1 / T)_{, \boldsymbol{x}}, \\
& \boldsymbol{J}^{2}=\boldsymbol{N}, \quad \boldsymbol{K}^{2}=\boldsymbol{B} / T, \\
& \boldsymbol{J}^{3}=R_{j i}, \quad \boldsymbol{K}^{3}=B_{i j} / T, \\
& \boldsymbol{J}^{4}=\boldsymbol{R}, \quad \boldsymbol{K}^{4}=-2 \boldsymbol{V} / T,
\end{aligned}
$$

where $T=\partial u / \partial s$ is the temperature, $\boldsymbol{B}=\boldsymbol{B}-\boldsymbol{B}^{\mathrm{e}}$ is the effective magnetic induction, $\boldsymbol{B}^{\mathrm{e}}=\partial u / \partial \boldsymbol{M}$ is the internal magnetic induction, $R_{i j}=R_{i j}^{\mathrm{s}}$ is the symmetric part of the magnetization flow tensor, $B_{i j}$ is the symmetric part of the tensor $B_{i, j}, \boldsymbol{R}=-(1 / 2) \boldsymbol{e}_{i} e_{i j k} R_{j k}^{\mathrm{a}}$ is a vector conjugated to the antisymmetric part of the magnetization flow tensor $R_{j k}^{\mathrm{a}}=-e_{i j k} R_{i}, \boldsymbol{V}=(1 / 2) \operatorname{rot} \boldsymbol{B}$ is the vortex of the effective magnetic induction.

According to the non-equilibrium thermodynamic principles generalized flows and forces are interrelated due to the constitutive relations $[4,5]$. Ignoring space dispersion the integral constitutive relations can be written in linear approximation in the form

$$
\boldsymbol{K}^{n}(t)=\int_{-\infty}^{t} I^{n m}\left(t, t^{\prime}\right) \boldsymbol{J}^{m}\left(t^{\prime}\right) \mathrm{d} t^{\prime}
$$

which allows for continuum memory and causality principle. The kinetic coefficients $I^{n m}$ (which depend on parameters $\boldsymbol{Q}$ including thermodynamic variables and 
flows) satisfy symmetry relations

$$
\begin{aligned}
& R^{\wedge} I_{i j \ldots}^{n m}\left(r^{\wedge} \boldsymbol{Q}\right)=I_{i^{\prime} j^{\prime} \ldots}^{n m}(\boldsymbol{Q}), \\
& c R^{\wedge} I_{i j \ldots}^{n m}\left(c^{Q} r^{\wedge} \boldsymbol{Q}\right)=I_{i^{\prime} j^{\prime} \ldots}^{n m}(\boldsymbol{Q}), \\
& c^{\prime} R^{\wedge} I_{j i \ldots}^{m n}\left(c^{\prime Q} r^{\wedge} \boldsymbol{Q}\right)=I_{i^{\prime} j^{\prime} \ldots}^{n m}(\boldsymbol{Q}), \\
& c c^{\prime} R^{\wedge} I_{j i \ldots}^{m n}\left(c^{Q} c^{\prime Q} r^{\wedge} \boldsymbol{Q}\right)=I_{i^{\prime} j^{\prime} \ldots}^{n m}(\boldsymbol{Q}),
\end{aligned}
$$

where $R^{\wedge} I_{i j \ldots}^{n m} \equiv\left(r_{i^{\prime} i} r_{j^{\prime} j \ldots}\right) I_{i j \ldots}^{n m}\left(r^{\wedge} \boldsymbol{Q}\right)$ are the coefficients transformation at simple rotations, $r^{\wedge}$ is the rotation operator, applied to each subscript, $r_{i^{\prime} i}$ is the rotation matrix; $c=c^{n} c^{m}= \pm 1, c=c, c^{\prime}, c^{\wedge} \boldsymbol{J}^{m}=c^{m} \boldsymbol{J}^{m}$ is the relationship that defines $c^{m}, c^{\wedge}=11^{\prime}$ are the space and time inversion operators, $c^{m}, c^{\prime m}=1$ for even type vectors $\boldsymbol{J}^{m}$ (axial $i$-vector, $\boldsymbol{J}^{2}$ ), $\underline{c}^{m}=-1, \mathrm{c}^{\prime m}=1$ for electric type vectors (polar $i$-vector, $\boldsymbol{J}^{3}, \boldsymbol{J}^{4}$ ), $\underline{c}^{m}=1$, $c^{\prime m}=-1$ for magnetic type vectors (axial $c$-vector), $\underline{c}^{m}=-1, c^{\prime m}=-1$ for magnetoelectric type vectors (polar $c$-vector, $\boldsymbol{J}^{1}$ ). The numbers $\underline{c}^{Q}$ and $c^{\prime}$ are determined in the same way as $c^{m}$ and $c^{\prime m}$. The generalized flows are expressed through the generalized forces similarly to relations (5).

Permutation of subscripts in (6) is due to the causality principle. For a symmetric relative to $1^{\prime}$ continuum the third relation of $(6)$ provides the classic symmetric relation $c^{\prime} I_{j i}^{m n}(-\boldsymbol{M},-\boldsymbol{B})=I_{i j}^{n m}(\boldsymbol{M}, \boldsymbol{B})$, where $c^{\prime}=1$, if $\boldsymbol{J}^{n}$ and $\boldsymbol{J}^{m}$ change or do not change the sign simultaneously under the action of $1^{\prime}$ and $c^{\prime}=-1$, when only one of the vectors changes its sign [6]. As seen from the second equation of (6), in a symmetric relative to 1 continuum the flow $\boldsymbol{J}^{2}$ does not connect with other flows.

Let us consider a highly symmetric (centrosymmetrical isotropic) continuum at constant temperature. Taking into account symmetric relations (6) and expanding the flows in constitutive relations (5) in series at point $t$ and keeping two terms of the series, one can write constitutive relations in the form of relaxation equations

$$
\begin{aligned}
& \tau_{\mathrm{v}} \boldsymbol{N}_{, t}+\boldsymbol{N}=\nu \boldsymbol{B}, \\
& \tau_{\mathrm{t}} R_{j k, t}+R_{j k}=P_{j k}, \quad P_{j k}=P_{0 j k}-\delta_{j k} l_{\tau} \tau_{\mathrm{v}} R_{n n, t}, \\
& P_{0 j k}=2 \eta\left(B_{j k}-\delta_{j k} B_{n n} / 3\right)+\delta_{j k} \xi B_{n n}, \\
& \tau_{\mathrm{v}} R_{n n, t}+R_{n n}=3 \xi B_{n n}, \\
& \tau_{\mathrm{r}} \boldsymbol{R}, \boldsymbol{t}+\boldsymbol{R}=-2 \chi \boldsymbol{V},
\end{aligned}
$$

where $\tau_{\mathrm{v}}$ is the relaxation time of volume force moment, $\tau_{\mathrm{t}}, \tau_{\mathrm{l}}, \tau_{\mathrm{v}}$ and $\tau_{\mathrm{r}}$ are the relaxation times for the symmetric and antisymmetric parts of the surface force moments $\left(\tau_{\mathrm{r}}, \tau_{\mathrm{t}}\right.$ and $\tau_{\mathrm{l}}$ determine relaxation of torsional, shear and longitudinal force moments, respectively), $\eta$, $\xi, \chi$ and $\nu$ are the coefficients of shear, volumetric, torsional and internal "magnetic viscosities" (they are positive due to positive-definite entropy production), $\tau_{\mathrm{v}}=$ $(3 \xi / 2 \eta)\left(\tau_{\mathrm{t}}+3 l \tau_{\mathrm{l}}\right)$ is the relaxation time for the trace of the force moment matrix, $l_{\tau}=l\left[\left(\tau_{\mathrm{l}} / \tau_{\mathrm{v}}\right)-1\right], l=I_{12}^{33} / 2 I_{44}^{33}$ is the coefficient which determines anisotropy of the continuum.

Equations (1) and (7) describe dynamics of the magnetization in linear approximation. Dependence of the coefficients on $T, E_{i}, M_{j}$ reduces to nonlinear terms in the constitutive equations. In particular, dependence of the linear viscosity coefficient on magnetization in the first equation of (7) results in the substitution for the volume force moment $\nu \boldsymbol{B} \rightarrow g[\boldsymbol{M} \boldsymbol{B}]+\left(\nu_{11}+\left(\nu_{12}+\nu_{44}\right) \boldsymbol{M}^{2}\right) \boldsymbol{B}-$ $\nu_{44}[\boldsymbol{M}[\boldsymbol{M B}]]$. Therefore, for a highly symmetric locally equilibrium $\left(\tau_{\mathrm{t}}, \tau_{\mathrm{r}}, \tau_{\mathrm{v}} \rightarrow 0\right)$ continuum Eq. (1) takes the form of the standard equation of motion for the magnetic moment vector [1], if one leaves the surface force moments out of consideration $(\chi, \eta, \xi \rightarrow 0)$.

Making use of (1) and implying constant coefficients in (7) the equation of motion for the magnetic moment vector can be written as

$\tau_{\mathrm{t}} \boldsymbol{M}_{, t t}+\boldsymbol{M}_{, t}+\eta \Delta \boldsymbol{B}=-\operatorname{grad} \varphi^{B}+\boldsymbol{N}^{\mathrm{e}}-\operatorname{rot} \boldsymbol{R}^{\mathrm{e}},(8)$ where $\Delta$ is the Laplace operator, $\varphi^{B}=\eta^{\mathrm{e}} B_{j j}+l_{\tau} R_{j j}$ is the effective scalar potential of the force moment, $\eta^{\mathrm{e}}=\left[(1 / 3) \eta+\xi\left(1-3 l_{\tau}\right)\right]$ is the effective magnetic viscosity coefficient, $R_{j j}=\left(3 \xi / \tau_{\mathrm{v}}\right) \int_{-\infty}^{t} B_{j j}\left(t^{\prime}\right) \exp \left(\left(t^{\prime}-\right.\right.$ t) $\left./ \tau_{\mathrm{v}}\right) \mathrm{d} t^{\prime}$ is the component of the potential, $\boldsymbol{N}^{\mathrm{e}}=\boldsymbol{N}+$ $\tau_{\mathrm{t}} \boldsymbol{N}_{\text {,t }}$ is the effective volume force moment, $\boldsymbol{N}(t)=$ $\left(\nu / \tau_{\mathrm{v}}\right) \int_{-\infty}^{t} \boldsymbol{B}\left(t^{\prime}\right) \exp \left(\left(t^{\prime}-t\right) / \tau_{\mathrm{v}}\right) \mathrm{d} t^{\prime}$ is the component of the force moment (the moment at a specified instant is determined by all the prehistory of the induction), $\boldsymbol{R}^{\mathrm{e}}=\boldsymbol{R}+\tau_{\mathrm{t}} \boldsymbol{R}_{t}$ is the effective vector potential, the curl of which yields the force moment, $\boldsymbol{R}(t)=$ $\left(-2 \chi / \tau_{\mathrm{r}}\right) \int_{-\infty}^{t} \boldsymbol{V}\left(t^{\prime}\right) \exp \left(\left(t^{\prime}-t\right) / \tau_{\mathrm{r}}\right) \mathrm{d} t^{\prime}$ is determined by the change in the field vortex for all past time.

In linear approximation $\boldsymbol{B}^{\mathrm{e}}=a \boldsymbol{M}$ and, accordingly, the internal energy produced by magnetization is $u_{\mathrm{m}}=$ $(1 / 2) a M^{2}$. Therefore, the energy $u_{\mathrm{m}}$ should be conceived as the spin-spin interaction energy (exchange energy) and the coefficient $a$ is as the interaction constant [6, 7]. For a locally equilibrium state $\left(\tau_{\mathrm{t}}, \tau_{\mathrm{v}} \rightarrow 0\right)$ with small inhomogeneity of the magnetic induction Eq. (8) describes diffusion of the magnetization. If the inhomogeneity of magnetization is also small, then from (8), evidently, the vector $\boldsymbol{M} \rightarrow \boldsymbol{B} / \mathbf{a}$ with a characteristic time of relaxation $\tau_{\mathrm{m}}=(a \nu)^{-1}$. For a locally non-equilibrium continuum the parabolic equation of magnetization diffusion is transformed into a hyperbolic one, allowing for solutions in the form of transverse and longitudinal waves.

Equations of motion for the vectors of polarization and electric current density have a form similar to (8).

At constant polarization and in the case of the Lorentz gauge the magnetic vector potential $(\boldsymbol{B}=\operatorname{rot} \boldsymbol{A})$ obeys the equation

$$
c^{-2} \boldsymbol{A}_{, t t}-\Delta \boldsymbol{A}=4 \pi \operatorname{rot} \boldsymbol{M} .
$$

The fields $\boldsymbol{A}(t, \boldsymbol{r})$ and $\boldsymbol{M}(t, \boldsymbol{r})$ are interrelated through the field vortexes $\operatorname{rot} \boldsymbol{M}$ and $\operatorname{rot} \boldsymbol{A}$, respectively.

\section{Coupled waves of the vector potential and magnetization}

Let us consider harmonic oscillations $\boldsymbol{M}, \boldsymbol{A} \propto$ $\exp (-i \omega t)$, where $\omega=\omega^{\prime}+i \omega^{\prime \prime}$ is the complex frequency, $\omega^{\prime}$ and $\omega^{\prime \prime}$ are the real and imaginary parts of $\omega$. In this case at constant coefficients of the "magnetic viscosity" Eqs. (8) and (9) take the form 
$-\Delta \boldsymbol{A}-k_{0}^{2} \boldsymbol{A}=4 \pi \operatorname{rot} \boldsymbol{M}$,

$c_{\mathrm{l}} \operatorname{grad} \operatorname{div} \boldsymbol{M}-\Delta \boldsymbol{M}-k_{\mathrm{m}}^{2} \boldsymbol{M}=k_{\mathrm{c}}^{2} \operatorname{rot} \boldsymbol{A}$,

where $k_{0}^{2}=(\omega / c)^{2}$ is the asymptotic wave number, $k_{\mathrm{m}}^{2}=c_{1} / c_{0}$ and $k_{\mathrm{c}}^{2}=c_{2} / c_{0}$ are the characteristic wave numbers, $c_{1}=1-a \eta_{1} / c_{0}, \eta_{1}=(4 / 3) \eta+\xi\left(1-3 l_{\tau \omega}\right)$ is the effective "magnetic viscosity", $l_{\tau \omega}=l_{\tau}\left[-\mathrm{i} \omega \tau_{\mathrm{v}} /\left(1-\mathrm{i} \omega \tau_{\mathrm{v}}\right)\right]$ is the coefficient which determines the viscosity frequency dependence, $c_{1}=\omega^{2} \tau_{\mathrm{t}}+\mathrm{i} \omega-a \nu \zeta_{\mathrm{v}}, c_{2}=(\omega / c)^{2}(\eta+$ $\left.\chi \zeta_{\mathrm{m}}\right)+\nu \zeta_{\mathrm{v}}, c_{0}=a_{\pi}\left(\eta+\chi \zeta_{\mathrm{m}}\right)$ are the coupling coefficients for the continuum and field, $\zeta_{\mathrm{m}}=\left(1-\mathrm{i} \omega \tau_{\mathrm{t}}\right) /\left(1-\mathrm{i} \omega \tau_{\mathrm{r}}\right)$ and $\zeta_{\mathrm{v}}=\left(1-\mathrm{i} \omega \tau_{\mathrm{t}}\right) /\left(1-\mathrm{i} \omega \tau_{\mathrm{v}}\right)$ are the functions which determine the frequency dependence of the coefficients, $a_{\pi}=a-4 \pi$ is the reciprocal quasi-static susceptibility.

Physical meaning of the coefficients results from the relation $\boldsymbol{M}=(\kappa / \mu) \boldsymbol{B}$, where $\kappa$ is the magnetic susceptibility, $\mu=1+4 \pi \kappa$ is the magnetic permeability [6]. As seen from the second formula of (10) for homogeneous magnetization the ratio $\kappa / \mu=-c_{2} / c_{1}$ and, consequently, $\kappa=-\left(\left(c_{1} / c_{2}\right)+4 \pi\right)^{-1}$. In the limit $\omega \rightarrow 0$ the quasi-static susceptibility $\kappa_{0}^{\prime}=1 / a_{\pi}$, for a paramagnet $\kappa_{0}^{\prime}>0$ and for a diamagnets $\kappa_{0}^{\prime}<0$. In the limit $\omega \rightarrow \infty$ susceptibility $\kappa_{\infty}^{\prime} \rightarrow-\gamma_{0} /\left(1+4 \pi \gamma_{0}\right)$, where $\gamma_{0}=\gamma_{\eta}+\gamma_{\chi}$, $\gamma_{\eta}=v_{\eta}^{2} / c^{2}$ and $\gamma_{\chi}=v_{\chi}^{2} / c^{2}$ are the ratios of the representative velocities and velocity of light, $v_{\eta}^{2}=\eta / \tau_{\mathrm{t}}$ and $v_{\chi}^{2}=\chi / \tau_{\mathrm{r}}$ are the velocities of the magnetization waves caused by shear and torsional "magnetic viscosities". If the low-frequency quasi-static susceptibility does not depend on non-equilibrium parameters of the continuum, the high-frequency asymptotic susceptibility is determined by the coefficients of the magnetic viscosity and relaxation times of the surface force moments.

Let elementary excitations be traveling plane waves of the form $\boldsymbol{A}, \boldsymbol{M} \propto \exp (\mathrm{i} k x-\mathrm{i} \omega t)$, where $\boldsymbol{k}$ is the wave vector. It follows from (10) that the wave components are related through

$$
\begin{aligned}
& \left(k^{2}-k_{0}^{2}\right) \boldsymbol{A}=\mathrm{i} 4 \pi[\boldsymbol{k} \boldsymbol{M}], \\
& \left(k^{2}-k_{\mathrm{m}}^{2}\right) \boldsymbol{M}-c_{\mathrm{l}} \boldsymbol{k}(\boldsymbol{k} \boldsymbol{M})=\mathrm{i} k_{\mathrm{c}}^{2}[\boldsymbol{k} \boldsymbol{A}] .
\end{aligned}
$$

The uncoupled longitudinal magnetization waves are described by the dispersion relation $k^{2}=k_{\mathrm{m}}^{2} /\left(1-c_{\mathrm{l}}\right)$. The transverse waves are coupled waves of vector potential and magnetization with the dispersion relation

$$
\left(q^{2}-\Omega^{2}\right)\left(q^{2}-q_{\mathrm{m}}^{2}\right)-4 \pi q_{\mathrm{c}}^{2} q^{2}=0,
$$

where $q^{2} \equiv \boldsymbol{q}^{2}=\boldsymbol{k}^{2}\left(c \tau_{\mathrm{m}}\right)^{2}, q_{\mathrm{m}, \mathrm{c}}^{2} \equiv k_{\mathrm{m}, \mathrm{c}}^{2}\left(c \tau_{\mathrm{m}}\right)^{2}$ are normalized wave numbers, $\Omega=\omega \tau_{\mathrm{m}}$ is the normalized frequency. Because of the isotropy of the continuum relation (12) does not depend on the direction of wave propagation. When $4 \pi q_{\mathrm{c}}^{2} \rightarrow 0$ the dispersion relation for coupled waves decomposes into dispersion relations for uncoupled waves of magnetization field $q_{1}^{2}=q_{\mathrm{m}}^{2}$ and EM field $q_{2}^{2}=\Omega^{2}$.

\section{Spectrum of locally non-equilibrium fluctuations}

Let us consider fluctuations of magnetization (wave packets) consisting of plane waves with real wave numbers and complex frequencies.
Equation (12) can be written as

$$
\begin{aligned}
& a_{06} \Omega^{6}+a_{04} \Omega^{4}+a_{02} \Omega^{2}+a_{00} \\
& \quad+\mathrm{i} \Omega\left(a_{05} \Omega^{4}+a_{03} \Omega^{2}+a_{01}\right)=0,
\end{aligned}
$$

where $a_{06}=\theta_{\mathrm{v}} \theta_{1}, a_{05}=\theta_{\mathrm{v}} \theta_{0}+\theta_{1}, a_{04}=a_{040}+a_{041} q^{2}$, $a_{040}=-\theta_{\mathrm{v}}-\theta_{0}-\theta_{1}, a_{041}=-a_{06}\left(1+a \gamma_{0}\right), a_{03}=a_{030}+$ $a_{031} q^{2}, a_{030}=-1-\theta_{0}, a_{031}=-a_{05}-a \gamma_{01}, a_{02}=1+$ $a_{021} q^{2}+a_{022} q^{4}, a_{021}=\theta_{\mathrm{v}}+\theta_{0}+\alpha \theta_{1}+a \gamma_{1}, a_{022}=a_{06} \alpha a \gamma_{0}$, $a_{01}=a_{011} q^{2}+a_{012} q^{4}, a_{011}=1+\alpha \theta_{0}, a_{012}=\alpha a \gamma_{01}$, $a_{00}=a_{001} q^{2}+a_{002} q^{4}, a_{001}=-\alpha, a_{002}=-\alpha a \gamma_{1}$ are coefficients, $\theta_{\mathrm{v}}=\tau_{\mathrm{v}} / \tau_{\mathrm{m}}, \theta_{\mathrm{t}}=\tau_{\mathrm{t}} / \tau_{\mathrm{m}}, \theta_{\mathrm{r}}=\tau_{\mathrm{r}} / \tau_{\mathrm{m}}$ are normalized relaxation times, $\theta_{0}=\theta_{\mathrm{t}}+\theta_{\mathrm{r}}, \theta_{1}=\theta_{\mathrm{t}} \theta_{\mathrm{r}}$ are the characteristic times of relaxation, $\gamma_{1}=\gamma_{\eta} \theta_{\mathrm{t}}+\gamma_{\chi} \theta_{\mathrm{r}}$, $\gamma_{01}=\gamma_{0} \theta_{1}+\gamma_{1} \theta_{\mathrm{v}}$ are the characteristic velocity ratios.

The real part of (13) is

$$
a_{3} \Omega^{\prime 6}+a_{2} \Omega^{\prime 4}+a_{1} \Omega^{\prime 2}+a_{0}=0,
$$

where $a_{3}=a_{06}, a_{2}=a_{04}-5 a_{05} \Omega^{\prime \prime}-15 a_{06} \Omega^{\prime \prime 2}, a_{1}=$ $a_{02}-3 a_{03} \Omega^{\prime \prime}-6 a_{04} \Omega^{\prime \prime 2}+10 a_{05} \Omega^{\prime \prime 3}+15 a_{06} \Omega^{\prime \prime 4}, a_{0}=$ $a_{00}-a_{01} \Omega^{\prime \prime}-a_{02} \Omega^{\prime \prime 2}+a_{03} \Omega^{\prime \prime 3}+a_{04} \Omega^{\prime \prime 4}-a_{05} \Omega^{\prime \prime 5}-a_{06} \Omega^{\prime \prime 6}$ are coefficients.

The imaginary part of (13) is

$$
b_{2} \Omega^{\prime 4}+b_{1} \Omega^{\prime 2}+b_{0}=0 \text {, }
$$

where $b_{2}=a_{05}+6 a_{06} \Omega^{\prime \prime}, b_{1}=a_{03}+4 a_{04} \Omega^{\prime \prime}-10 a_{05} \Omega^{\prime \prime 2}-$ $20 a_{06} \Omega^{\prime \prime 3}, b_{0}=a_{01}+2 a_{02} \Omega^{\prime \prime}-3 a_{03} \Omega^{\prime \prime 2}-4 a_{04} \Omega^{\prime \prime 3}+$ $5 a_{05} \Omega^{\prime \prime 4}+6 a_{06} \Omega^{\prime \prime 5}$ are coefficients.

If $b_{2} \neq 0, \mathrm{Eq} .(15)$ provides the dispersion relation for spin-electromagnetic (SE) and electromagnetic-spin (ES) modes in an implicit form

$$
\Omega_{1,2}^{\prime 2}=-\left(b_{1} / 2 b_{2}\right) \pm\left[\left(b_{1} / 2 b_{2}\right)^{2}-\left(b_{0} / b_{2}\right)\right]^{1 / 2} .
$$

Substitution of (16) into (14) gives the equation for the imaginary part of the frequency in an implicit form

$$
i_{0} I_{0}+i_{1} I_{1}+a_{3}^{2} I_{2}=0,
$$

where $i_{0}=a_{0} b_{1}-a_{1} b_{0}, I_{0}=b_{2}\left(2 a_{3} b_{0}+a_{2} b_{1}-a_{1} b_{2}\right)-a_{3} b_{1}^{2}$, $i_{1}=a_{0} b_{2}-a_{2} b_{0}, I_{1}=b_{2} i_{1}+a_{3} b_{0} b_{1}, I_{2}=b_{0}^{3}$ are functions of the coefficients. Inserting the coefficients from (14) and (15) in (17) one can write it in the explicit form

$$
\sum_{n=0}^{n=15} e_{n} \Omega^{\prime \prime n}=0
$$

where $e_{n}=\sum_{m, l}^{n=m+l} i_{0 m} I_{0 l}+\sum_{p, r}^{n=p+r} i_{1 p} I_{l r}+a_{06}^{2} I_{2 n}$ are coefficients which depend on $q^{2}, 0 \leq m \leq 9$, $0 \leq l \leq 6,0 \leq p \leq 7,0 \leq r \leq 8$ are integers, $i_{00}=a_{03} a_{00}-a_{02} a_{01}, i_{01}=4 a_{04} a_{00}+2 a_{03} a_{01}-2 a_{02}^{2}$, $i_{02}=-10 a_{05} a_{00}+2 a_{04} a_{01}+8 a_{03} a_{02}, i_{03}=-20 a_{06} a_{00}+$ $12 a_{04} a_{02}-8 a_{03}^{2}, i_{04}=5 a_{06} a_{01}-15 a_{05} a_{02}-25 a_{04} a_{03}$, $i_{05}=-16 a_{06} a_{02}+34 a_{05} a_{03}-20 a_{04}^{2}, i_{06}=42 a_{06} a_{03}+$ $56 a_{05} a_{04}, i_{07}=72 a_{06} a_{04}-40 a_{05}^{2}, i_{08}=-105 a_{06} a_{05}$, $i_{09}=-70 a_{06}^{2}$ are the coefficients at powers $\Omega^{\prime \prime}$ of the function $i_{0}, I_{00}=a_{06}\left(2 a_{05} a_{01}-a_{03}^{2}\right)+a_{05}\left(a_{04} a_{03}-\right.$ $\left.a_{05} a_{02}\right), \quad I_{01}=a_{06}\left(12 a_{06} a_{01}-8 a_{05} a_{02}-2 a_{04} a_{03}\right)+$ $a_{05}\left(4 a_{04}^{2}-2 a_{05} a_{03}\right), I_{02}=a_{06}\left(-12 a_{06} a_{02}+5 a_{05} a_{03}+\right.$ $\left.8 a_{04}^{2}\right)-24 a_{05}^{2} a_{04}, I_{03}=a_{06}\left(22 a_{06} a_{03}-116 a_{05} a_{04}\right)+$ $40 a_{05}^{3}, I_{04}=a_{06}\left(325 a_{05}^{2}-152 a_{06} a_{04}\right), I_{05}=932 a_{06}^{2} a_{05}$, $I_{06}=932 a_{06}^{3}$ are the coefficients at powers $\Omega^{\prime \prime}$ of the function $I_{0}, i_{10}=a_{05} a_{00}-a_{04} a_{01}, i_{11}=6 a_{06} a_{00}+$ $4 a_{05} a_{01}-2 a_{04} a_{02}, i_{12}=9 a_{06} a_{01}+9 a_{05} a_{02}+3 a_{04} a_{03}$, 
$i_{13}=24 a_{06} a_{02}-14 a_{05} a_{03}+4 a_{04}^{2}, i_{14}=-39 a_{06} a_{03}-$ $24 a_{05} a_{04}, i_{15}=-60 a_{06} a_{04}+24 a_{05}^{2}, i_{16}=98 a_{06} a_{05}$, $i_{17}=84 a_{06}^{2}$ are the coefficients at powers $\Omega^{\prime \prime}$ of the function $i_{1}, I_{10}=a_{06} a_{03} a_{01}+a_{05}\left(a_{05} a_{00}-a_{04} a_{01}\right), I_{11}=$ $a_{06}\left(12 a_{05} a_{00}-2 a_{04} a_{01}+2 a_{03} a_{02}\right)+a_{05}\left(4 a_{05} a_{01}-2 a_{04} a_{02}\right)$, $I_{12}=a_{06}\left(36 a_{06} a_{00}+23 a_{05} a_{01}-4 a_{04} a_{02}-3 a_{03}^{2}\right)+$ $a_{05}\left(9 a_{05} a_{02}+3 a_{04} a_{03}\right), I_{13}=a_{06}\left(34 a_{06} a_{01}+58 a_{05} a_{02}+\right.$ $\left.2 a_{04} a_{03}\right)+a_{05}\left(-14 a_{05} a_{03}+4 a_{04}^{2}\right), I_{14}=a_{06}\left(104 a_{06} a_{02}-\right.$ $\left.88 a_{05} a_{03}+8 a_{04}^{2}\right)-24 a_{05}^{2} a_{04}, I_{15}=-a_{06}\left(-168 a_{06} a_{03}-\right.$ $\left.144 a_{05} a_{04}\right)+24 a_{05}^{3}, I_{16}=a_{06}\left(-256 a_{06} a_{04}+192 a_{05}^{2}\right)$, $I_{17}=512 a_{06}^{2} a_{05}, I_{18}=384 a_{06}^{3}$ are the coefficients at powers $\Omega^{\prime \prime}$ of the function $I_{1}, I_{20}=a_{01}^{3}, I_{21}=6 a_{01}^{2} a_{02}$, $I_{22}=a_{01}\left(-9 a_{01} a_{03}+12 a_{02}^{2}\right), I_{23}=-12 a_{01}\left(a_{01} a_{04}+\right.$ $\left.3 a_{02} a_{03}\right)+8 a_{02}^{3}, \quad I_{24}=a_{01}\left(15 a_{01} a_{05}-48 a_{02} a_{04}+\right.$ $\left.27 a_{03}^{2}\right)-36 a_{02}^{2} a_{03}, \quad I_{25}=a_{01}\left(18 a_{01} a_{06}+60 a_{02} a_{05}+\right.$ $\left.72 a_{03} a_{04}\right)+a_{02}\left(-48 a_{02} a_{04}+54 a_{03}^{2}\right), I_{26}=a_{01}\left(72 a_{02} a_{06}-\right.$ $\left.90 a_{03} a_{05}+48 a_{04}^{2}\right)+a_{02}\left(60 a_{02} a_{05}+144 a_{03} a_{04}\right)-27 a_{03}^{3}$, $I_{27}=a_{01}\left(-108 a_{03} a_{06}-120 a_{04} a_{05}\right)+a_{02}\left(72 a_{02} a_{06}-\right.$ $\left.180 a_{03} a_{05}+96 a_{04}^{2}\right)-108 a_{03}^{2} a_{04}, I_{28}=a_{01}\left(-144 a_{04} a_{06}+\right.$ $\left.75 a_{05}^{2}\right)+a_{02}\left(-216 a_{03} a_{06}-240 a_{04} a_{05}\right)+a_{03} 144\left(a_{03} a_{05}-\right.$ $\left.a_{04}^{2}\right), I_{29}=a_{01} 180 a_{05} a_{06}+a_{02}\left(-288 a_{04} a_{06}+150 a_{05}^{2}\right)+$ $a_{03}\left(162 a_{03} a_{06}+360 a_{04} a_{05}\right)-64 a_{04}^{3}, I_{210}=a_{01} 108 a_{06}^{2}+$ $360 a_{02} a_{05} a_{06}+a_{03}\left(432 a_{04} a_{06}-225 a_{05}^{2}\right)+240 a_{04}^{2} a_{05}$, $I_{211}=216 a_{02} a_{06}^{2}-540 a_{03} a_{05} a_{06}+a_{04}\left(288 a_{04} a_{06}-\right.$ $\left.300 a_{05}^{2}\right), \quad I_{212}=-324 a_{03} a_{06}^{2}-720 a_{04} a_{05} a_{06}+125 a_{05}^{3}$, $I_{213}=-432 a_{04} a_{06}^{2}+450 a_{05}^{2} a_{06}, I_{214}=540 a_{05} a_{06}^{2}, I_{215}=$ $216 a_{06}^{3}$ are the coefficients at powers $\Omega^{\prime \prime}$ of the function $I_{2}$. When $\theta_{\mathrm{v}}=0$ the fifteenth-order Eq. (18) reduces to the tenth-order one.

Generally (18) can have up to fifteenth solutions $\Omega^{\prime \prime}\left(q^{2}\right)$. Each solution provides up to two spectral branches (16) (dissipation splits the spectrum). The domain of existence of excitations is determined by the condition $b_{1}^{2}-4 b_{0} b_{2} \geq 0$, and it should be noted that because of positive left side of (16), when $b_{0} b_{2}>0$ there are two solutions for $b_{1} b_{2}<0$ and there are no solutions for $b_{1} b_{2}>0$; when $b_{0} b_{2}<0$ there is one solution. Hence, in a locally non-equilibrium continuum propagation of the excitations will be complex. As (18) has no analytical solution, let us begin with a qualitative analysis of the equation.

Along with null solution $\Omega_{00}^{\prime \prime}=0$ at the point $q=0$ there are also nonzero solutions, satisfying the equation $\sum_{n=1}^{n=15} e_{n 0} \Omega_{0}^{\prime \prime n-1}=0$, where $e_{n 0}=e_{n}(q=0)$. The frequencies of homogeneous excitations are determined from (15) at $q=0$ and $\Omega^{\prime \prime}=\Omega_{0 j}^{\prime \prime}$. Particularly, frequencies of homogeneous continuous oscillations are $\Omega_{20}^{\prime}=\left[\left(1+\theta_{0}\right) /\left(\theta_{\mathrm{v}} \theta_{0}+\theta_{1}\right)\right]^{1 / 2}$ and $\Omega_{10}^{\prime}=0$. ES branch has an energy gap $\Omega_{20}^{\prime}$, which is determined by relaxation times. The gap results from dynamic interaction of electromagnetic and spin subsystems. Slow magnetization produces an effective restoring force for rapid vector potential, setting a finite oscillation frequency. SE branch has no gap. The "null" frequency oscillations are due to translation invariance of the continuum (absence of the restoring force). However in this case nonlinear effects are essential [8]. As a result the frequency will depend on the amplitude and the oscillations of "null" frequency will have "null" amplitude.

In the high wave number region $\left(q^{2} \rightarrow \infty\right)$ at the restricted values of $\Omega^{\prime \prime}$ in (18) the basic coefficients are $q^{12}$ and Eq. (18) reduces to the third degree equation $\sum_{n=0}^{n=3} e_{n 6} \Omega_{\infty}^{\prime \prime} n=0$ with constant coefficients. In the low $q$-region the number of solutions can be greater than that in high $q$-region. In the case of constant damping the linear dispersion relation results from $(15)$ as $\Omega_{1,2}^{\prime}=v_{1,2} q$, where $v_{1,2}=\left[-\left(b_{11} / 2 b_{20}\right) \pm\right.$ $\left.\left(\left(b_{11} / 2 b_{20}\right)^{2}-\left(b_{02} / b_{20}\right)\right)^{1 / 2}\right]^{1 / 2}$ are the asymptotic velocities, $b_{20}=a_{05}+6 a_{06} \Omega_{\infty}^{\prime \prime}, b_{11}=a_{031}+4 a_{041} \Omega_{\infty}^{\prime \prime}$, $b_{02}=a_{012}+2 a_{022} \Omega_{\infty}^{\prime \prime}$. If $\left|4 b_{20} b_{02} / b_{11}^{2}\right| \ll 1$, then $v_{1}=\left(-b_{02} / b_{11}\right)^{1 / 2}, v_{2}=\left(-b_{11} / b_{20}\right)^{1 / 2}$. Each solution $\Omega_{\infty}^{\prime \prime}$ results in up to two branches $\Omega^{\prime}(q)$. For low dumping $\Omega_{\infty}^{\prime \prime} \ll\left(a_{05} / 6 a_{06}\right),\left(\gamma_{01} / 4 a_{06} \gamma_{0}\right)$ the coefficients $b_{20}=a_{05}, b_{11}=-a_{05}-a \gamma_{01}, b_{02}=\alpha a \gamma_{01}$ do not depend on $\Omega_{\infty}^{\prime \prime}$ and the branches are joined.

There are critical wave numbers $q_{\mathrm{c}}$ satisfying the equation $\Omega^{\prime \prime}\left(q_{\mathrm{c}}^{2}\right)=0$. At the point $q_{\mathrm{c}}$ the imaginary part of $\Omega^{\prime \prime}$ changes its sign. Amplitudes of the excitations with $\Omega^{\prime \prime}<0$ and $\Omega^{\prime \prime}>0$ decrease or increase with time, respectively. Wave numbers $q_{\mathrm{c}}$ are the points of the loss of stability for the zero field state. Nonzero solutions $q_{\mathrm{c}}$ satisfy the equation $\sum_{n=1}^{n=6} e_{0 n} q_{\mathrm{c}}^{2(n-1)}=0$. In paramagnets this equation has no solutions as all coefficients have the same sign. In diamagnets there are at least two solutions $q_{\mathrm{c}}^{2}$ as $e_{01}, e_{06}<0$ regardless of the sign of $\alpha$. At the points $q_{\mathrm{c}}$, along with the zero solution, there are solutions satisfying the equation $\sum_{n=1}^{n=15} e_{n c} \Omega_{c}^{\prime \prime n-1}=0$, where $e_{n c}=e_{n}\left(q_{c}^{2}\right)$.

Let us consider the case of small dumping. The quadratic approximation of (18) gives

$$
\Omega_{0,1}^{\prime \prime}\left(q^{2}\right)=-\left(e_{1} / 2 e_{2}\right) \pm\left[\left(e_{1} / 2 e_{2}\right)^{2}-\left(e_{0} / e_{2}\right)\right]^{1 / 2},
$$
where $e_{1}^{2}-4 e_{0} e_{2} \geq 0$. If $e_{0} e_{2}<0$ then $\Omega_{0}^{\prime \prime}>0$ and $\Omega_{1}^{\prime \prime}<0$, if $e_{0} e_{2}>0$ then $\Omega_{0,1}^{\prime \prime}>0$ for $e_{1} e_{2}<0$ and $\Omega_{0,1}^{\prime \prime}<0$ for $e_{1} e_{2}>0$. At the points $q_{\mathrm{c}}$ the coefficient $e_{0}=0$ and so $\Omega_{0}^{\prime \prime}\left(q_{\mathrm{c}}\right)=0, \Omega_{1}^{\prime \prime}\left(q_{\mathrm{c}}\right)=-e_{1} / e_{2}$.

In the high wave number region (19) becomes $\Omega_{0,1 \infty}^{\prime \prime}=\left(e_{16} / 2 e_{26}\right)\left[-1 \pm\left(1-\left(4 e_{26} e_{06} / e_{16}^{2}\right)\right)^{1 / 2}\right]$, where $e_{26}=-\left(2 a_{06}^{2} \alpha a \gamma_{0}\right)^{2} a_{05}\left[\left(1+a \gamma_{0}\right)^{2}-4 \alpha a \gamma_{0}\right], e_{16}=$ $2\left(a_{06} \alpha a\right)^{2}\left(j_{01} \gamma_{0} \gamma_{161}+a_{06} \gamma_{01} \gamma_{162}\right), \gamma_{161}=a_{06}(\alpha-1) \gamma_{0}+$ $(1+4 \alpha) \gamma_{01}, \gamma_{162}=\gamma_{01}\left(1+a \gamma_{0}\right)^{2}-2 \alpha a \gamma_{0}\left(\gamma_{01}+a_{05} \gamma_{0}\right)$, $e_{06}=(\alpha-1) \alpha^{2} a \gamma_{01} j_{01}^{2}$. For $4 e_{26} e_{06} / e_{16}^{2} \ll 1$ asymptotic attenuations $\Omega_{0 \infty}^{\prime \prime}=-e_{06} / e_{16}$ and $\Omega_{1 \infty}^{\prime \prime}=-e_{16} / e_{26}$ do not depend on the wave number. Substitution of the constants $\Omega_{0,1 \infty}^{\prime \prime}$ into (15) gives the linear dispersion relation

$$
\Omega_{1,2}^{\prime}=v_{1,2 \infty} q
$$

where $v_{1,2 \infty}=\left[-\left(b_{1 \infty} / 2 b_{2 \infty}\right) \pm\left(\left(b_{1 \infty} / 2 b_{2 \infty}\right)^{2}-\right.\right.$ $\left.\left.\left(b_{0 \infty} / b_{2 \infty}\right)\right)^{1 / 2}\right]^{1 / 2}$ are the asymptotic velocities of $\mathrm{SE}$ and ES modes, $b_{2 \infty}=a_{05}+6 a_{06} \Omega_{0,1 \infty}^{\prime \prime}$, $b_{1 \infty}=a_{031}+4 a_{041} \Omega_{0,1 \infty}^{\prime \prime}, \quad b_{0 \infty}=-a_{012}-$ $2 a_{022} \Omega_{0,1 \infty}^{\prime \prime}$. For low dumping $\left|\Omega_{0,1 \infty}^{\prime \prime}\right| \ll a_{05} / 6 a_{06}$, $\left(a_{05}+a \gamma_{01}\right) / 4 a_{06}\left(1+a \gamma_{0}\right), \quad \gamma_{01} / 2 a_{06} \gamma_{0}$ the velocities $v_{2,1 \infty}=\left[(1 / 2)(1+u)\left(1 \pm\left(1-4 \alpha u /(1+u)^{2}\right)^{1 / 2}\right]^{1 / 2}\right.$, 
where $u=a \gamma_{01} / a_{05}$. There are two branches in paramagnets $(\alpha>0)$ and one ES branch in diamagnets $(\alpha<0)$. For $4 \alpha u /(1+u)^{2} \ll 1 \mathrm{SE}$ and $\mathrm{ES}$ wave velocities are $v_{1 \infty}=[\alpha u /(1+u)]^{1 / 2}$ and $v_{2 \infty}=(1+u)^{1 / 2}$, respectively.

In the small wave number region (19) gives $\Omega_{0}^{\prime \prime}=$ $-e_{0} / e_{1}$ and $\Omega_{1}^{\prime \prime}=-e_{1} / e_{2}$ for $4 e_{0} e_{2} / e_{1}^{2} \ll 1$. The dispersion relations for SE mode $\Omega_{1}^{\prime 2}=-b_{0} / b_{1}$ and ES mode $\Omega_{2}^{\prime 2}=-b_{1} / b_{2}$ result from (16) on condition $4 b_{0} b_{2} / b_{1}^{2} \ll 1$, where $b_{0}=a_{01}+2 a_{02} \Omega_{0,1}^{\prime \prime}, b_{1}=$ $a_{03}+4 a_{04} \Omega_{0,1}^{\prime \prime}, b_{2}=a_{05}+6 a_{06} \Omega_{0,1}^{\prime \prime}$. In the following the analysis is limited by holding only terms of the order of $q^{4}$.

Dumping of the dominant mode is determined by the function $\Omega_{0}^{\prime \prime}=\Omega_{01}^{\prime \prime} q^{2}+\Omega_{02}^{\prime \prime} q^{4}$, where $\Omega_{01}^{\prime \prime}=-e_{01} / e_{10}$, $\Omega_{02}^{\prime \prime}=-\left(e_{10} e_{02}-e_{01} e_{11}\right) / e_{10}^{2}$ are the coefficients of the function, $e_{01}=(\alpha-1) I_{000}, e_{02}=(\alpha-1)\left[I_{001}-\left(\alpha \theta_{0} \theta_{1}-\right.\right.$ $\left.\left.a \gamma_{1}\right) I_{000}-\theta_{1} I_{101}+\theta_{v 0}\left(\theta_{0} j_{00}-a_{05}^{2}\right)\right]$ and $e_{10}=-2 I_{000}$, $e_{11}=(\alpha-1) I_{010}-2 I_{001}+i_{011} I_{000}+2 j_{00}\left[i_{101}+\theta_{+}(1+\right.$ $\left.\left.\alpha \theta_{0}\right)\right]-2 \alpha \theta_{+} a_{05}^{2}$ are the coefficients at wave numbers in $e_{0}$ and $e_{1}, I_{000}=\left(1+\theta_{0}\right) j_{00}-a_{05}^{2}>0, I_{001}=a_{05}\left[a_{06}(1+\right.$ $\left.\left.\alpha \theta_{0}\right)+(1-\alpha) \theta_{1}^{2}-a_{05} a \gamma_{1}\right]+a \gamma_{01} j_{00}+\left(1+\theta_{0}\right) j_{01}, I_{101}=$ $\left(1+\alpha \theta_{0}\right) j_{00}-\alpha a_{05}^{2}, I_{010}=-2 a_{05}\left(4 a_{06}+j_{20}\right)+2 \theta_{+} j_{00}$ are the coefficients at wave numbers in $I_{00}, I_{10}$ and $I_{01}, \theta_{+}=$ $\theta_{\mathrm{v}}+\theta_{0}+\theta_{1}, j_{00}=\theta_{0}\left(\theta_{\mathrm{v}}^{2}+a_{05}\right)+\theta_{1}^{2}, j_{20}=-a_{05}\left(1+\theta_{0}\right)-\theta_{+}^{2}$, $i_{011}=4(\alpha-1)\left(\theta_{\mathrm{v}}+\theta_{0}\right)-2\left(1+\theta_{0}\right)\left(1+\alpha \theta_{0}\right)-4 a \gamma_{1}$ are the components of the coefficients. When $q \rightarrow 0$ the dumping $\Omega_{0}^{\prime \prime}=-(2 \pi / a) q^{2}$ does not depend on non-equilibrium parameters and $\Omega_{0}^{\prime \prime} \rightarrow 0$ as the wave number squared. The point of the loss of stability of the dominant mode $q_{\mathrm{c}}^{2}=-\Omega_{01}^{\prime \prime} / \Omega_{02}^{\prime \prime}$ exists if $\Omega_{02}^{\prime \prime}>0$.

The dispersion relation for the dominant SE mode is

$$
\Omega_{10}^{\prime 2}=v_{10}^{2} q^{2}+v_{11}^{4} q^{4},
$$

where $v_{10}=(\alpha)^{1 / 2}$ is the velocity in the locally equilibrium continuum, $v_{11}^{4}=\left(1+\theta_{0}\right)^{-1}\left[-\alpha\left(a_{05}+(1-\right.\right.$ $\left.\left.\alpha)\left(\theta_{\mathrm{v}}-\theta_{1}\right)\right)+(1-\alpha)\left(1+\theta_{0}+(1-\alpha)\left(I_{j} / I_{000}\right)\right)\right]$ is the coefficient determining dispersion of the velocity, $I_{j}=j_{00} \theta_{+}+2 a_{05}\left[a_{05}\left(1+\theta_{0}\right)+\theta_{+}^{2}-4 a_{06}\right]$. In the region $q^{2} \ll\left|v_{10}^{2} / v_{11}^{4}\right|$ the dispersion relation is a linear function $\Omega_{1}^{\prime}=v_{10} q$. Thus for $q \rightarrow 0$ and $q \rightarrow \infty$ the dominant $\mathrm{SE}$ mode exists in paramagnets and does not exist in diamagnets. Nevertheless in diamagnets the dominant mode can exist for wave numbers, lying in narrow intervals due to positive $v_{11}^{4}$.

The dispersion relation for the dominant ES mode is

$$
\Omega_{20}^{\prime 2}=\Omega_{200}^{\prime 2}+v_{21}^{2} q^{2}+v_{22}^{4} q^{4}
$$

where $\Omega_{200}^{\prime 2}=\left(1+\theta_{0}\right) / a_{05}$ is the energy gap, $v_{21}^{2}=$ $1+u+\left[(1-\alpha) / a_{05}^{2}\right]\left(a_{05} \theta_{+}-3 j_{00}\right)$ is square of the characteristic velocity, $v_{22}^{4}=(1-\alpha) a_{06}\left[3 v_{21}^{2}+\left(\Omega_{020}^{\prime \prime} / a_{06}\right)\left(\theta_{+}-\right.\right.$ $\left.\left.\left(3 j_{00} / a_{05}\right)\right)+(1-\alpha)\left(3 / a_{05}^{2}\right)-2\left(1+a \gamma_{0}\right)\right]$ is the coefficient which determines velocity dispersion, $\Omega_{020}^{\prime \prime}=$ $2 \alpha \theta_{\mathrm{v}}-1-a \gamma_{1}+(\alpha-1)\left[\theta_{\mathrm{v}}+2 \theta_{0}+\left(I_{j} / I_{000}\right)\right]$. Thus the dominant ES mode exists in paramagnets and diamagnets for all wave numbers.

Dumping of the first mode is determined by the function $\Omega_{1}^{\prime \prime}=\Omega_{10}^{\prime \prime}+\Omega_{11}^{\prime \prime} q^{2}+\Omega_{12}^{\prime \prime} q^{4}$, where $\Omega_{10}^{\prime \prime}=-e_{10} / e_{20}$, $\Omega_{11}^{\prime \prime}=\left(e_{10} e_{21}-e_{11} e_{20}\right) / e_{20}^{2}, \Omega_{12}^{\prime \prime}=\left(e_{10} e_{22}-e_{12} e_{20}-\right.$ $\left.e_{21} e_{20} \Omega_{11}^{\prime \prime}\right) / e_{20}^{2}$ are the coefficients of the function, $e_{1 n}$ and $e_{2 n}$ are the coefficients at $q^{2 n}$ in $e_{1}$ and $e_{2}$. For $q \rightarrow 0$ dumping is $\Omega_{1}^{\prime \prime} \rightarrow \Omega_{10}^{\prime \prime}=-1 /\left[4\left(1+\theta_{0}\right)+2 a_{05}\left(a_{05}\left(1+\theta_{0}\right)+\right.\right.$ $\left.\left.\theta_{+}^{2}-4 a_{06}\right) I_{000}^{-1}\right]$, for the first mode the point of the stability loss is $q_{\mathrm{c} 1,2}^{2}=\left[-\Omega_{11}^{\prime \prime} \pm\left(\Omega_{11}^{\prime \prime}{ }^{2}-4 \Omega_{10}^{\prime \prime} \Omega_{12}^{\prime \prime}\right)^{1 / 2}\right] / 2 \Omega_{12}^{\prime \prime}$. There are two values of $q_{\mathrm{c} 1,2}^{2}$ for $\Omega_{12}^{\prime \prime}, \Omega_{11}^{\prime \prime}<0$ and one value of $q_{\mathrm{c} 1}^{2}$ for $\Omega_{12}^{\prime \prime}>0$.

The dispersion relation for the first SE mode is determined by the function $\Omega_{11}^{\prime}=\left(\Omega_{110}^{\prime 2}+v_{11}^{2} q^{2}+v_{12}^{4} q^{4}\right)^{1 / 2}$, where $\Omega_{110}^{\prime 2}=-b_{00} / b_{10}, v_{11}^{2}=\left(b_{00} b_{11}-b_{01} b_{10}\right) / b_{1} 0^{2}$, $v_{11}^{4}=\left(b_{00} b_{12}-b_{02} b_{10}-b_{01} b_{10} v_{11}^{2}\right) / b_{1} 0^{2}$ are the coefficients of the function, $b_{00}=2 \Omega_{10}^{\prime \prime}, b_{01}=1+\alpha \theta_{0}+2\left(\theta_{\mathrm{s}}+a \gamma_{1}\right)$, $b_{02}=\alpha a \gamma_{1}+2\left[a_{06} \alpha a \gamma_{0} \Omega_{10}^{\prime \prime}+\left(\theta_{\mathrm{s}}+a \gamma_{1}\right) \Omega_{11}^{\prime \prime}+\Omega_{12}^{\prime \prime}\right]$, $b_{10}=-\left(1+\theta_{0}\right)-4 \theta_{+} \Omega_{10}^{\prime \prime}, b_{11}=-\left(a_{06}+a \gamma_{01}\right)-\left[a_{06}(1+\right.$ $\left.\left.a \gamma_{0}\right) \Omega_{10}^{\prime \prime}+\theta_{+} \Omega_{11}^{\prime \prime}\right], b_{12}=-4\left[a_{06}\left(1+a \gamma_{0}\right) \Omega_{11}^{\prime \prime}+\theta_{+} \Omega_{12}^{\prime \prime}\right]$, $b_{20}=a_{05}+6 a_{06} \Omega_{10}^{\prime \prime}, b_{21}=6 a_{06} \Omega_{11}^{\prime \prime}, b_{22}=6 a_{06} \Omega_{12}^{\prime \prime}$ are components of the coefficients, $\theta_{\mathrm{s}}=\theta_{\mathrm{v}}+\theta_{0}+\alpha \theta_{1}$. For $1+\theta_{0}>4 \theta_{+} \Omega_{10}^{\prime \prime}$ the dispersion relation can have an impulse gap as $\Omega_{110}^{\prime 2}<0$.

The dispersion relation for the first ES mode is $\Omega_{21}^{\prime}=$ $\left(\Omega_{210}^{\prime 2}+v_{21}^{2} q^{2}+v_{22}^{4} q^{4}\right)^{1 / 2}$, where $\Omega_{210}^{\prime 2}=-b_{10} / b_{20}$ is the energy gap, $v_{21}^{2}=\left(b_{10} b_{21}-b_{11} b_{20}\right) / b_{20}^{2}, v_{22}^{4}=\left(b_{10} b_{22}-\right.$ $\left.b_{12} b_{20}-b_{21} b_{20} v_{21}^{2}\right) / b_{10}^{2}$ are the characteristic velocities.

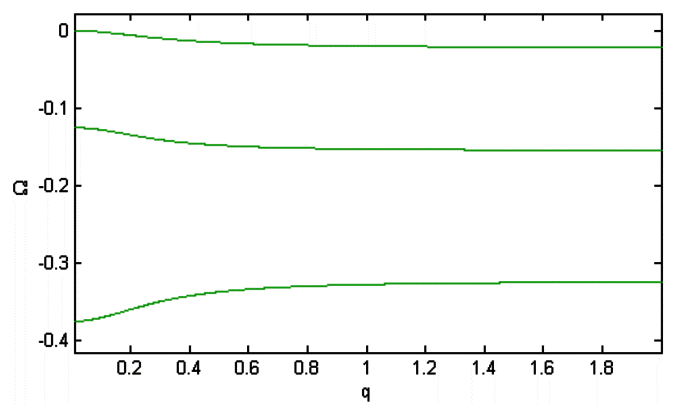

Fig. 1. Dependence of imaginary part of the frequency on wave number in paramagnet.

Let us consider numerical solutions. Figure 1 shows $\Omega^{\prime \prime}(q)$ according to (17) for parameters $\theta_{\mathrm{v}}=4, \theta_{\mathrm{t}}=2$, $\theta_{\mathrm{r}}=4, \gamma_{\eta}=0.05, \gamma_{\chi}=0.07, a=10 \pi$. At $q \rightarrow 0$ the imaginary parts of the dominant and the first and second branches tend to zero and finite quantities, respectively. At $q \rightarrow \infty$ all branches tend to constants. Comparison of the exact numerical and quadratic approximation relations shows quantitative and qualitative agreement between the relations for the dominant and the first branches, respectively.

The imaginary part of (15) (a fifth order equation with regard to $\Omega^{\prime \prime}$ ) being expressed through special functions as $\Omega^{\prime \prime}\left(q^{2}\right)$ and inserted in (14) gives the dispersion relation $\Omega^{\prime 2}\left(q^{2}\right)$. Figure 2 shows the relations $\Omega^{\prime}(q)$ in quadratic in $\Omega^{\prime \prime}$ approximation for the same parameters as in Fig. 1. The ES branches have an energy gap and exhibit linear relation in the high wave number region. The dominant SE mode exists for all $q$ 's. The first SE mode has an impulse gap. The second SE mode exists for a small interval of wave numbers. 


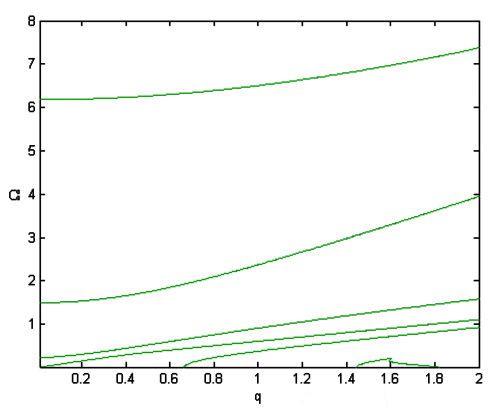

Fig. 2. Dependence of real part of the frequency on wave number in paramagnet.

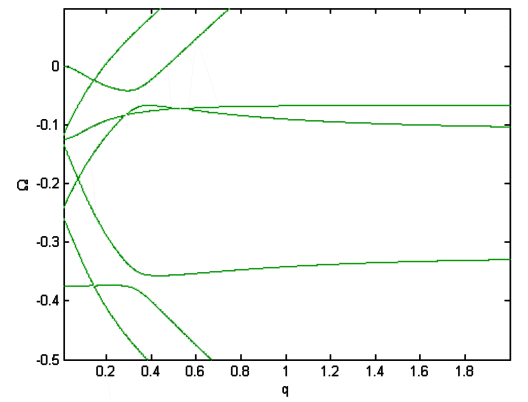

Fig. 3. Dependence of imaginary part of the frequency on wave number in diamagnets.

Figure 3 shows the relation $\Omega^{\prime \prime}(q)$ for $a=\pi$ while other parameters are as in the preceding figures. It can be seen that there are two unstable branches $\left(\Omega^{\prime \prime}>0\right)$. The dependence of the critical wave numbers on spin-spin interaction constant is shown in Fig. 4 . In the region $a>4 \pi$ the base state is stable. In the region $a<4 \pi$ there are two values of $q_{\mathrm{c}}$, which uniformly increase when $a \rightarrow 4 \pi$. The unstable region is restricted by space dispersion. The amplitude of excitations is restricted by nonlinear effects. Consequently, in diamagnets one can get continuous excitations which have zero total momentum and moment of momentum in accordance with the conservation laws. In the case $a<0$ there is one unstable branch in low wave number interval starting from $q=0\left(\Omega_{0}^{\prime \prime}=2 \pi q^{2} /|a|\right)$. Therefore the stable state is a ferromagnetic one.

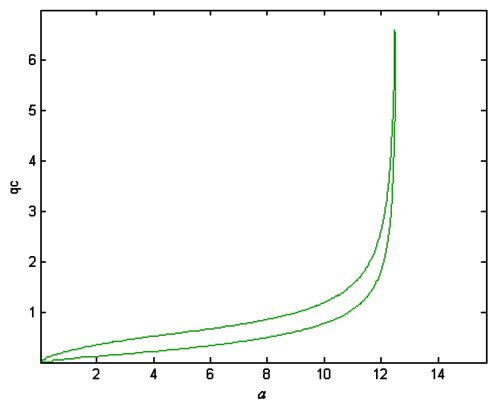

Fig. 4. Dependence of critical wave numbers on spin-spin interaction constant.
The spectrum of the locally equilibrium excitations has one dumping mode without any energy gap.

\section{Wave propagation in locally non-equilibrium continuum}

Let us consider wave excitation by an external source at the boundary of the continuum. In this case the frequency is real.

On the basis of (12) one can write squares of wave numbers of SE $(n=1)$ and $\operatorname{ES}(n=2)$ branches as

$$
q_{n}^{2}=\left[f_{\Omega} \pm\left(f_{\Omega m}^{2}\right)^{1 / 2}\right] / 2 f_{0},
$$

where $f_{\Omega m}^{2}=f_{\Omega}^{2}-4 \Omega^{2} f_{m 0}, f_{m 0}=f_{\mathrm{m}} f_{0}, f_{\mathrm{m}}=a_{06} \Omega^{4}+$ $a_{040} \Omega^{2}+1+\mathrm{i} \Omega\left(a_{05} \Omega^{2}+a_{030}\right), f_{0}=a_{022} \Omega^{2}+a_{002}+$ i $\Omega a_{012}, f_{\Omega}=a_{041} \Omega^{4}+a_{021} \Omega^{2}+a_{001}+\mathrm{i} \Omega\left(a_{031} \Omega^{2}+a_{011}\right)$ are the functions of frequency.

Separating the real and imaginary parts of the above functions one can write $q_{n}^{2}=\left(F_{n}^{\prime}+\mathrm{i} F_{n}^{\prime \prime}\right) / 2\left(f_{0}^{\prime}+\right.$ $\left.\mathrm{i} f_{0}^{\prime \prime}\right)$, where $F_{1,2}^{\prime}=f_{\Omega}^{\prime} \pm\left(\left(f_{\Omega m}^{2}\right)^{1 / 2}\right)^{\prime}$ and $F_{1,2}^{\prime \prime}=$ $f_{\Omega}^{\prime \prime} \pm\left(\left(f_{\Omega m}^{2}\right)^{1 / 2}\right)^{\prime \prime}$ are the real and imaginary parts, $\left(\left(f_{\Omega m}^{2}\right)^{1 / 2}\right)^{\prime, \prime \prime}=2^{-1 / 2}\left[\left(\left|f_{\Omega m}^{2}\right|^{2}\right)^{1 / 2} \pm f_{\Omega m}^{2}\right]^{1 / 2}$ are the real and imaginary parts of the root of the function $f_{\Omega m}^{2}$, $\left|f_{\Omega m}^{2}\right|^{2}=\left(f_{\Omega m}^{2}{ }^{\prime}\right)^{2}+\left(f_{\Omega m}^{2}{ }^{\prime \prime}\right)^{2}, f_{\Omega m}^{2}{ }^{\prime}=f_{\Omega}^{\prime 2}-f_{\Omega}^{\prime \prime 2}-4 \Omega^{2} f_{m 0}^{\prime}$ and $f_{\Omega m}^{2}{ }^{\prime \prime}=2 f_{\Omega}^{\prime} f_{\Omega}^{\prime \prime}-4 \Omega^{2} f_{m 0}^{\prime \prime}$ is the square of the module, real and imaginary parts of the radicand, $f_{m 0}^{\prime}=f_{m}^{\prime} f_{0}^{\prime}-$ $f_{\mathrm{m}}^{\prime \prime} f_{0}^{\prime \prime}$ and $f_{m 0}^{\prime \prime}=f_{\mathrm{m}}^{\prime} f_{0}^{\prime \prime}+f_{\mathrm{m}}^{\prime \prime} f_{0}^{\prime}$ are the real and imaginary parts of $f_{m 0}$. Hence $q_{n}^{2}=q_{n}^{2 \prime}+\mathrm{i} q_{n}^{2 \prime \prime}$, where $q_{n}^{2 \prime}=$ $\left(F_{n}^{\prime} f_{0}^{\prime}+F_{n}^{\prime \prime} f_{0}^{\prime \prime}\right) / 2\left|f_{0}\right|^{2}$ and $q_{n}^{2 \prime \prime}=\left(F_{n}^{\prime \prime} f_{0}^{\prime}-F_{n}^{\prime} f_{0}^{\prime \prime}\right) / 2\left|f_{0}\right|^{2}$ are the real and imaginary parts of the squares of wave numbers, $\left|f_{0}\right|^{2}=f_{0}^{\prime 2}+f_{0}^{\prime \prime 2}$.

As a result relations determining dispersion and dumping for SE and ES modes take a form

$$
q_{n}^{\prime \prime \prime \prime}=2^{-1 / 2}\left\{\left[\left(q_{n}^{2 \prime}\right)^{2}+\left(q_{n}^{2 \prime \prime}\right)^{2}\right]^{1 / 2} \pm q_{n}^{2 \prime}\right\}^{1 / 2}
$$

Relations (24) contain a discrete set of characteristic frequencies $\Omega_{l 1}, \Omega_{l 2}$, which satisfy the equations $q_{1}^{2 \prime}\left(\Omega_{l 1}\right)=0, q_{2}^{2 \prime}\left(\Omega_{l 2}\right)=0$, and frequencies $\Omega_{\mathrm{c} 1}, \Omega_{\mathrm{c} 2}$, which satisfy the equations $q_{1}^{2 \prime \prime}\left(\Omega_{\mathrm{c} 1}\right)=0, q_{2}^{2 \prime \prime}\left(\Omega_{\mathrm{c} 2}\right)=0$, respectively. At frequencies $\Omega_{l 1}, \Omega_{l 2}$ the real and imaginary parts of the wave numbers are $q_{l 1}=q_{1}^{\prime}=q_{1}^{\prime \prime}=$ $(1 / 2)\left|q_{1}^{2 \prime \prime}\left(\Omega_{l 1}\right)\right|^{1 / 2}, q_{l 2}=q_{2}^{\prime}=q_{2}^{\prime \prime}=(1 / 2)\left|q_{2}^{2 \prime \prime}\left(\Omega_{l 2}\right)\right|^{1 / 2}$, respectively. The boundary frequencies $\Omega_{l 1}, \Omega_{l 2}$ separate the regions of transmission and non-transmission, where $q_{n}^{\prime}>q_{n}^{\prime \prime}$ and $q_{n}^{\prime}<q_{n}^{\prime \prime}$, respectively. At frequencies $\Omega_{\mathrm{c} 1}$ and $\Omega_{\mathrm{c} 2}$ the imaginary part of wave numbers $q_{1}^{\prime \prime}\left(\Omega_{\mathrm{c} 1}\right)=0$ and $q_{2}^{\prime \prime}\left(\Omega_{\mathrm{c} 2}\right)=0$ in the case $q_{1}^{2 \prime \prime}\left(\Omega_{\mathrm{c} 1}\right)>0$ and $q_{2}^{2 \prime \prime}\left(\Omega_{\mathrm{c} 2}\right)>$ 0 . Waves of such frequencies (transparency frequencies) propagate at constant amplitude with wave numbers $q_{\mathrm{c} 1}^{\prime}=\left(q_{1}^{2 \prime}\left(\Omega_{\mathrm{c} 1}\right)\right)^{1 / 2}$ and $q_{\mathrm{c} 2}^{\prime}=\left(q_{2}^{2 \prime}\left(\Omega_{\mathrm{c} 2}\right)\right)^{1 / 2}$, respectively. In the case $q_{1}^{2 \prime}\left(\Omega_{\mathrm{c} 1}\right)<0$ and $q_{2}^{2 \prime}\left(\Omega_{\mathrm{c} 2}\right)<0$ the real parts of wave numbers $q_{1}^{\prime}\left(\Omega_{\mathrm{c} 1}\right)=0$ and $q_{2}^{\prime}\left(\Omega_{\mathrm{c} 2}\right)=0$. The waves of the frequencies $\Omega_{\mathrm{c} 1}$ and $\Omega_{\mathrm{c} 2}$ (non-transparency frequencies) do not penetrate into the continuum. These waves damp with a constant phase within the characteristic distances $\left|q_{1}^{2 \prime}\left(\Omega_{\mathrm{c} 1}\right)\right|^{-1 / 2}$ and $\left|q_{2}^{2 \prime}\left(\Omega_{\mathrm{c} 2}\right)\right|^{-1 / 2}$, and in the dumping region there is no energy flow $\left\langle\boldsymbol{J}^{\mathrm{f}}\right\rangle$.

The transparency effect can be interpreted in the following way. EM field induces locally non-equilibrium 
magnetization during a time smaller than relaxation time of the magnetization. The alternating magnetization causes radiation of EM waves in phase with EM field. As a result the field energy is conserved. This effect is similar to the self-induced transparency effect in optics, when a short pulse of coherent light (with duration shorter than relaxation time of the polarization) first excites atoms (transferring energy to them) and then stimulates atoms to radiate coherently (receiving the energy back). In the case of non-transparency the non-equilibrium magnetization radiates EM waves in antiphase to inducing oscillations of the field causing changes of the magnetization.

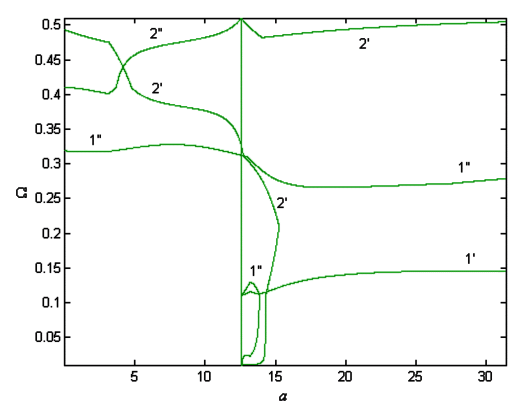

Fig. 5. Dependence of boundary frequencies and transparency (non-transparency) frequencies for SE $\left(1^{\prime}\right.$ and $\left.1^{\prime \prime}\right)$, ES $\left(2^{\prime}\right.$ and $\left.2^{\prime \prime}\right)$ branches on spin-spin interaction constant.

The results of numerical solutions of equations $q_{n}^{\prime}\left(\Omega_{l n}\right)=0$ and $q_{n}^{\prime \prime}\left(\Omega_{\mathrm{cn}}\right)=0$ are presented in Fig. 5 for parameters $\theta_{\mathrm{v}}=4, \theta_{\mathrm{t}}=2, \theta_{\mathrm{r}}=4, \gamma_{\eta}=0.05, \gamma_{\chi}=0.07$ in the interval $0.01 \pi \leq a \leq 10 \pi$. In diamagnets $(a<4 \pi)$ the SE branch has the non-transparency frequency across the whole region, ES branch has the boundary frequency and non-transparency frequency over nearly the first half of the region and the boundary frequency and transparency frequency over the second half of the region. In paramagnets $(a>4 \pi)$ the SE branch has a non-transparency frequency, the boundary frequency and two transparency frequencies in narrow region about $4 \pi$, ES branch has three frequencies. Beyond the mentioned region the $\mathrm{SE}$ branch has the boundary frequency and transparency frequency, ES branch has only the boundary frequency.

In general the analysis of relation (24) is a very complex problem. Let us consider some specific cases.

In the frequency region, where the first condition $\left(q_{n}^{2 \prime}\right)^{2} \gg\left(q_{n}^{2 \prime \prime}\right)^{2}$ is fulfilled, and particularly in the vicinity of $\Omega_{\mathrm{c} n}$, the dispersion and dumping for SE and ES modes are determined by

$$
\begin{aligned}
& q_{n}^{\prime \prime \prime \prime}=2^{-1 / 2}\left|q_{n}^{2 \prime}\right|^{1 / 2}\left[1 \pm \operatorname{sgn}\left(q_{n}^{2 \prime}\right)\right. \\
& \left.\quad+(1 / 2)\left(q_{n}^{2 \prime \prime} / q_{n}^{2 \prime}\right)^{2}\right]^{1 / 2} .
\end{aligned}
$$

The form of this relation depends on the sign of $q_{n}^{2 \prime}$. In the case $q_{n}^{2 \prime}>0$ the real part is $q_{n}^{\prime}=\left(q_{n}^{2 \prime}\right)^{1 / 2}$ and the imaginary part is $q_{n}^{\prime \prime}=\left|q_{n}^{2 \prime \prime}\right| / 2\left(q_{n}^{2 \prime}\right)^{1 / 2}$. In the case $q_{n}^{2 \prime}<0$ the real part is $q_{n}{ }^{\prime}=\left|q_{n}^{2 \prime \prime}\right| / 2\left(-q_{n}^{2 \prime}\right)^{1 / 2}$ and the imaginary part is $q_{n}^{\prime \prime}=\left(-q_{n}^{2 \prime}\right)^{1 / 2}$. When $q_{n}^{2 \prime}$ changes its sign the expressions determining the real and imaginary parts of the wave numbers change places. In this approximation in the vicinity of $\Omega_{\mathrm{cn}}$ in the first case the imaginary part and in the second case the real part are proportional to the deviation module of the wave frequency from frequencies of transparency or non-transparency: $q_{n}^{\prime \prime, \prime}=$ $q_{\Omega c n}^{\prime \prime}\left|\Omega-\Omega_{c n}\right|$, where $q_{\Omega c n}^{\prime \prime}=\left.\left(\left|\left(\partial q_{n}^{2 \prime \prime} / \partial \Omega\right)\right| / 2\left|q_{n}^{2 \prime}\right|^{1 / 2}\right)\right|_{\Omega c n}$ is calculated at the points $\Omega_{\mathrm{c} n}$.

In the frequency region, where the second condition $\left(q_{n}^{2 \prime}\right)^{2} \ll\left(q_{n}^{2 \prime \prime}\right)^{2}$ is fulfilled, and particularly, in the vicinity of $\Omega_{l n}$, the dispersion and dumping for SE and ES modes are determined by the relations

$$
q_{n}^{\prime, \prime \prime}=2^{-1 / 2}\left|q_{n}^{2 \prime \prime}\right|^{1 / 2}\left[1 \pm\left(q_{n}^{2 \prime} / 2\left|q_{n}^{2 \prime \prime}\right|\right)\right] .
$$

In the neighborhood of $\Omega_{l n}$ in linear approximation the real and imaginary parts are determined by $q_{n}^{\prime \prime}{ }^{\prime \prime}-$ $q_{l n}=(1 / 4)\left(q_{\Omega l n}^{\prime \prime} \pm q_{\Omega l n}^{\prime}\right)\left(\Omega-\Omega_{l n}\right)$, where $q_{l n}=$ $q_{n}^{\prime}\left(\Omega_{l n}\right)=q_{n}^{\prime \prime}\left(\Omega_{l n}\right)$ are the boundary frequency wave numbers, $q_{\Omega l n}^{\prime \prime}=\left.\left(\partial q_{n}^{2 \prime \prime} / \partial \Omega\right)\right|_{\Omega \ln } / q_{l n}$ and $q_{\Omega \ln }^{\prime}=$ $\left.\left(\partial q_{n}^{2 \prime} / \partial \Omega\right)\right|_{\Omega l n} / q_{l n}$ are determined by the derivatives at the points $\Omega_{l n}$.

For the both high and low frequency regions $\left(f_{\Omega m}^{2}\right)^{2} \gg$ $\left(f_{\Omega m}^{2}{ }^{\prime \prime}\right)^{2}$ as $f_{\Omega m}^{2}$ 'is a polynomial of the eighth degree with a term of zero degree and $f_{\Omega m}^{2}$ " is a polynomial of the seventh degree without a term of zero degree.

In the high frequency region for maximum degrees of $\Omega$ one can write $q_{n}^{2 \prime}=\Omega^{2} / v_{n}^{2}$, where $v_{n}^{2}=$ $2 \alpha a \gamma_{0} /\left[\left(1+a \gamma_{0}\right) \pm f_{\infty}^{\prime 1 / 2}\right]^{1 / 2}$ is the squared velocity, $f_{\infty}^{\prime}=\left(1+a \gamma_{0}\right)^{2}-4 \alpha a \gamma_{0}$ and $q_{n}^{2 \prime \prime}=\Omega \rho_{n}$, where $\rho_{1,2}=$ $\left[\gamma_{2} \theta_{\mathrm{v}} \pm\left(\gamma_{0}\left|f_{\infty}^{\prime \prime}\right|\left(f_{\infty}^{\prime}\right)^{-1 / 2}-\gamma_{01}\left(f_{\infty}^{\prime}\right)^{1 / 2}\right)\right] / 2 \alpha a \gamma_{0}^{2} \theta_{\mathrm{v}} \theta_{1}, f_{\infty}^{\prime \prime}=$ $a \gamma_{01}\left(1-2 \alpha+a \gamma_{0}\right)+\left(\theta_{\mathrm{v}} \theta_{0}+\theta_{1}\right)\left(1+(1-2 \alpha) a \gamma_{0}\right)$. For $\Omega^{2} \gg v_{n}^{4} \rho_{n}^{2}$ the first condition is fulfilled. For paramagnets for SE and ES waves and for diamagnets for ES waves $q_{n}^{2 \prime}$ is positive. On the basis of (25) dispersion of these waves is described by a linear function

$$
q_{n}^{\prime}=\Omega / v_{n},
$$

where $v_{n}=\left|v_{n}^{2}\right|^{1 / 2}$ are the asymptotic velocities, for $(1+$ $\left.a \gamma_{0}\right)^{2} \gg\left|4 \alpha a \gamma_{0}\right|$ the velocities $v_{1}=\left[\alpha a \gamma_{0} /\left(1+a \gamma_{0}\right)\right]^{1 / 2}$ and $v_{2}=\left(1+a \gamma_{0}\right)^{1 / 2}$. When $\gamma_{0} \rightarrow 0$ the velocity of SE wave $v_{1} \rightarrow 0$ and the velocity of ES wave $v_{2} \rightarrow 1$. Dumping of these waves is described by relations $q_{n}^{\prime \prime}=v_{n}\left|\rho_{n}\right| / 2$ bearing no dependence on frequency. The ratio of imaginary and real parts decreases with increase of frequency as $\Omega^{-1}$. For diamagnets for SE waves $q_{n}^{2 \prime}$ is negative and, hence, the expressions for real and imaginary parts of such waves exchange: $q_{n}^{\prime}=v_{n}\left|\rho_{n}\right| / 2$ and $q_{n}^{\prime \prime}=\Omega / v_{n}$. High-frequency $\mathrm{SE}$ waves do not propagate in diamagnets in contrast to ES waves.

Taking into account the terms of the order of $\Omega^{2}$ one can write $q_{n}^{2 \prime}=\left(\Omega^{2}-\Omega_{0 n}^{2}\right) / v_{0 n}^{2}$ and $q_{n}^{2 \prime \prime}=\Omega \rho_{0 n}$ for the low frequency region, where $\Omega_{0 n}^{2}=v_{0 n}^{2}(1 \pm|\alpha| / \alpha) / 2 a \gamma_{1}$ is the characteristic frequency, $v_{0 n}^{2}=2 \alpha a \gamma_{1}^{2} / g_{n}$ is the characteristic velocity squared, $g_{n}=g_{n 0}+g_{n 1}+g_{n 2}, g_{n 0}=$ $\left(\theta_{\mathrm{v}} \theta_{1} \gamma_{0}+\theta_{\mathrm{v} \gamma} \gamma_{1}\right)(\alpha \pm|\alpha|), \theta_{\mathrm{v} \gamma}=\theta_{\mathrm{v}}^{2}+\left(\theta_{1} \gamma_{0} / \gamma_{1}\right)^{2}, g_{n 1}=$ $\gamma_{01}\left(-a_{011} \pm\left|a_{011}\right|\right), g_{n 2}=\gamma_{1}\left(\theta_{\mathrm{s}}+a \gamma_{1} \pm|\alpha|\left(-f_{\Omega m 1}^{2}{ }^{\prime} / 2 \alpha^{2}\right)\right)$, $f_{\Omega m 1}^{2}{ }^{\prime}=2 \alpha\left(a \gamma_{1}-\theta_{\mathrm{s}}\right)-a_{011}^{2}, \theta_{\mathrm{s}}=\theta_{\mathrm{v}}+\theta_{0}+\alpha \theta_{1}, \rho_{0 n}=$ $-\left[\gamma_{1}\left(-a_{011} \pm\left|a_{011}\right|\right)+\gamma_{01}(\alpha \pm|\alpha|)\right] / 2 \alpha a \gamma_{1}^{2}$. For paramagnetics and SE waves $q_{1}^{2 \prime}=\left(\Omega^{2}-\Omega_{01}^{2}\right) / v_{01}^{2}$ and $q_{1}^{2 \prime \prime}=$ 
$-\Omega \gamma_{01} / a \gamma_{1}^{2}$, where $\Omega_{01}^{2}=v_{01}^{2} / a \gamma_{1}, v_{01}^{2}=2 \alpha \gamma_{1}^{2} /\left(g_{10}+\right.$ $\left.g_{12}\right), g_{10}=2 \alpha\left(\theta_{\mathrm{v}} \theta_{1} \gamma_{0}+\theta_{\mathrm{v} \gamma} \gamma_{1}\right), g_{12}=2 \theta_{\mathrm{s}}+a_{011}^{2} / 2 \alpha$, and for $\mathrm{ES}$ waves $q_{2}^{2 \prime}=\Omega^{2} / v_{02}^{2}$ and $q_{2}^{2 \prime \prime}=\Omega a_{011} / \alpha a \gamma_{1}$, where $v_{02}^{2}=2 \alpha \gamma_{1}^{2} /\left(g_{21}+g_{22}\right), g_{21}=-2 \gamma_{01} a_{011}, g_{22}=2 a \gamma_{1}-$ $a_{011}^{2} / 2 \alpha$. For diamagnets and SE waves $q_{1}^{2 \prime}=\Omega^{2} / v_{01}^{2}$ and $q_{1}^{2 \prime \prime}=\Omega a_{011} / \alpha a \gamma_{1}$ for $a_{011}<0, q_{1}^{2 \prime \prime}=0$ for $a_{011}>0$, where $v_{01}^{2}=2 \alpha \gamma_{1}^{2} /\left(g_{11}+g_{12}\right), g_{11}=0$ for $a_{011}<0$, $g_{11}=-2 \gamma_{01} a_{011}$ for $a_{011}>0, g_{12}=2 a \gamma_{1}-a_{011}^{2} / 2 \alpha$, and for ES waves $q_{2}^{2 \prime}=\left(\Omega^{2}-\Omega_{\mathrm{s} 2}^{2}\right) / v_{02}^{2}$ and $q_{2}^{2 \prime \prime}=-\Omega \gamma_{01} / a \gamma_{1}^{2}$ for $a_{011}<0, q_{2}^{2 \prime \prime}=-\Omega\left(\alpha \gamma_{01}+f_{\Omega 1} \gamma_{1}\right) / \alpha a \gamma_{1}^{2}$ for $a_{011}>0$, where $\Omega_{02}^{2}=v_{02}^{2} / a \gamma_{1}, v_{02}^{2}=2 \alpha \gamma_{1}^{2} / g_{2}, g_{2}=g_{20}+g_{21}+g_{22}$, $g_{20}=2\left(\theta_{\mathrm{v}} \theta_{1} \gamma_{0}+\theta_{\mathrm{v} \gamma} \gamma_{1}\right), g_{21}=-2 \gamma_{01} a_{011}$ for $a_{011}<0$, $g_{21}=0$ for $a_{011}>0, g_{22}=2 \theta_{\mathrm{s}}+a_{011}^{2} / 2 \alpha$.

Let us consider SE waves in paramagnets and ES waves in diamagnets. Then $q_{1,2}^{2}{ }^{\prime} \rightarrow-1 / a \gamma_{1}, q_{1,2}^{2}{ }^{\prime \prime} \rightarrow 0$ when $\Omega \rightarrow 0$ and, therefore, the first condition is satisfied. In virtue of $(25)$ one can write

$$
\begin{aligned}
& q_{n}^{\prime}=\Omega\left|\Omega^{2}-\Omega_{0 n}^{2}\right|^{-1 / 2} v_{0 n}\left|\rho_{0 n}\right|, \\
& q_{n}^{\prime \prime}=\left|\Omega^{2}-\Omega_{0 n}^{2}\right|^{1 / 2} / v_{0 n},
\end{aligned}
$$

where $v_{0 n}=\left|v_{0 n}^{2}\right|^{1 / 2}$. In the region $\Omega^{2} \ll \Omega_{0 n}^{2}$ dispersion and dumping are described by a linear function and constant. When $\Omega^{2} \rightarrow \Omega_{0 n}^{2}$ for the waves the second condition is satisfied. On the basis of (26) one can write

$$
q_{n}^{\prime, \prime \prime}=\Omega^{1 / 2}\left|\rho_{0 n} / 2\right|^{1 / 2}\left[1 \pm\left(\Omega^{2}-\Omega_{0 n}^{2}\right) / 2 \Omega\left|\rho_{0 n}\right| v_{0 n}^{2}\right] .
$$

The same sort of root dependence of the real and imaginary parts of the wave number on frequency determines the skin-effect. Difference of the real and imaginary parts changes sign under transition of $\Omega$ through $\Omega_{0 n}$. For frequencies $\Omega^{2} \gg \Omega_{0 n}^{2}, v_{0 n}^{4} / \rho_{0 n}^{2}$ the first condition is satisfied again. Therefore, for $v_{0 n}^{2}<0$ the dispersion and dumping of the waves are determined by relations (28). For $v_{0 n}^{2}>0$ the formulae for the real and imaginary parts in (28) exchange.

Let us consider ES waves in paramagnets and SE waves in diamagnets. For these waves the second condition is satisfied at $\Omega \rightarrow 0$ and, hence, the dispersion and dumping of the waves are determined by (29). For frequencies $\Omega^{2} \gg\left(v_{0 n}^{2} a_{011} / \alpha a \gamma_{1}\right)^{2}$ the first condition is satisfied. Consequently, for $v_{0 n}^{2}<0$ the dispersion and dumping of the waves are determined by (28). For $v_{0 n}^{2}>0$ formulae for the real and imaginary parts in (28) exchange.

The results of numerical solutions of Eqs. (24) are presented in Fig. 6 for parameters $\theta_{\mathrm{v}}=4, \theta_{\mathrm{t}}=2, \theta_{\mathrm{r}}=4$, $\gamma_{\eta}=0.05, \gamma_{\chi}=0.07, a=10 \pi$. It can be seen that the SE branch has both the boundary and transparency frequencies. The value $q_{1}^{\prime \prime}(\Omega=0)$ calculated from (28) coincides with that on the curve $1^{\prime \prime}$. In the low frequency range the ES branch exhibits the skin-effect and boundary frequency. In the high frequency range the dispersion relations are linear functions of frequency, dumping is constant.

Figure 7 shows the real and imaginary parts of the wave numbers as functions of frequency for diamagnets for $a=2 \pi$ and the other parameters are the same as in Fig. 6. The SE branch has a non-transparency fre-

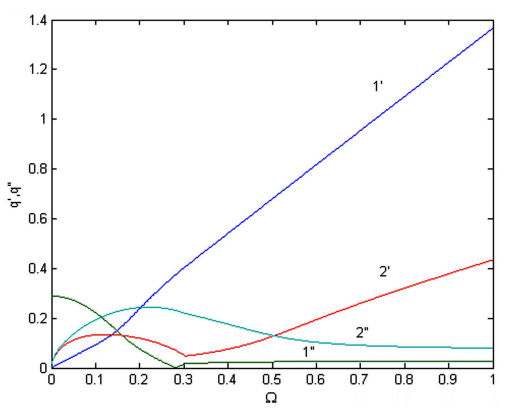

Fig. 6. Dependence of real, imaginary parts of wave number for SE $\left(1^{\prime}, 1^{\prime \prime}\right)$ and ES $\left(2^{\prime}, 2^{\prime \prime}\right)$ branches on frequency in paramagnet.

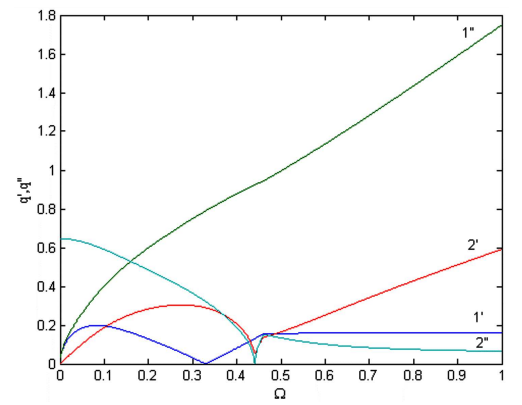

Fig. 7. Dependence of real, imaginary parts of wave number for SE $\left(1^{\prime}, 1^{\prime \prime}\right)$ and $\mathrm{ES}\left(2^{\prime}, 2^{\prime \prime}\right)$ branches on frequency in diamagnets.

quency. The ES branch has boundary frequency and a transparency frequency. The value $q_{2}^{\prime \prime}(\Omega=0)$ calculated from (28) coincides with the value that on the curve $2^{\prime \prime}$. In the frequency range below the boundary frequency ES waves do not propagate. In the high frequency range ES waves have linear dispersion and constant dumping that complies with the result of analytic calculation.

In locally equilibrium continuum waves have none of the above features except for the boundary frequency in diamagnets.

\section{Standing waves in locally non-equilibrium continuum}

Let us consider localized oscillations. On the basis of the system of Eqs. (10) one can write the equations for vector potential in the form

$$
\Delta\left(\Delta \boldsymbol{A}+\left(k_{0}^{2}+k_{m \mathrm{c}}^{2}\right) \boldsymbol{A}\right)+k_{0}^{2} k_{\mathrm{m}}^{2} \boldsymbol{A}=0,
$$

and magnetization in the form

$$
\begin{gathered}
\Delta\left(\Delta \boldsymbol{M}+\left(k_{0}^{2}+k_{m \mathrm{c}}^{2}\right) \boldsymbol{M}\right)+k_{0}^{2} k_{\mathrm{m}}^{2} \boldsymbol{M} \\
-\operatorname{grad} \operatorname{div}\left(c_{\mathrm{l}} \Delta \boldsymbol{M}+c_{k} \boldsymbol{M}\right)=0,
\end{gathered}
$$

where $k_{m \mathrm{c}}^{2}=k_{\mathrm{m}}^{2}+4 \pi k_{\mathrm{c}}^{2}, c_{k}=c_{\mathrm{l}} k_{0}^{2}+4 \pi k_{\mathrm{c}}^{2}$.

Let the continuum be of the form of a parallelepiped with sides $l_{1}, l_{2}, l_{3}$, point peculiarities of the fields be absent $(\operatorname{div} \boldsymbol{A}=0, \operatorname{div} \boldsymbol{M}=0)$ and the fields be zero on the boundary surface. Then the solution of (30) can be 
sought

$$
\begin{aligned}
& A_{x}=A_{1} \cos k_{1} x \sin k_{2} y \sin k_{3} z, \\
& A_{y}=A_{2} \sin k_{1} x \cos k_{2} y \sin k_{3} z, \\
& A_{z}=A_{3} \sin k_{1} x \sin k_{2} y \cos k_{3} z,
\end{aligned}
$$

where $k_{1}=\left(\pi / l_{1}\right) n, k_{2}=\left(\pi / l_{2}\right) m, k_{3}=\left(\pi / l_{3}\right) p$, $n, m, p=0,1, \ldots$ are integers. The potential amplitudes are related by $A_{1} k_{1}+A_{2} k_{2}+A_{3} k_{3}=0$. The natural frequencies, as seen from (30) and (31), satisfy the equation

$$
q_{\nu}^{4}-q_{\nu}^{2}\left(q_{0}^{2}+q_{\mathrm{m}}^{2}+4 \pi q_{\mathrm{c}}^{2}\right)+q_{0}^{2} q_{\mathrm{m}}^{2}=0,
$$

where $q_{\nu}^{2} \equiv q_{n m p}^{2}=q_{1}^{2}+q_{2}^{2}+q_{3}^{2}$ are the discrete wave numbers, $q_{i} \equiv k_{i}\left(c \tau_{\mathrm{m}}\right)$. Equation (33) is transformed into (12) at substitution of discrete wave number by a continuous wave number $\left(q_{\nu}^{2} \rightarrow q^{2}\right)$. Hence the dispersion and dumping of the standing waves are determined by the system of Eqs. (16), (18) on the replacement $q^{2} \rightarrow q_{\nu}^{2}$.

Making use of (18) and taking into account the above indicated conformities one can write the equation for the imaginary part of frequency

$$
\sum_{n=0}^{n=15} e_{\nu n} \Omega_{\nu}^{\prime \prime}=0,
$$

where $e_{\nu n}=e_{n}\left(q^{2}=q_{\nu}^{2}\right)$. Inserting (34) into (16) with replacement $b_{1,2}$ by $b_{\nu 1}, 2=b_{1,2}\left(q^{2}=q_{\nu}^{2}, \Omega^{\prime \prime}=\Omega_{\nu}^{\prime \prime}\right)$ gives the expression for frequencies of standing modes in the form

$$
\begin{aligned}
& \left(\Omega^{\prime 2}{ }_{\nu}^{2}\right)_{1,2}=-\left(b_{\nu 1} / 2 b_{\nu 2}\right) \\
& \quad \pm\left[\left(b_{\nu 1} / 2 b_{\nu 2}\right)^{2}-\left(b_{\nu 0} / b_{\nu 2}\right)\right]^{1 / 2} .
\end{aligned}
$$

Equation (35) has real solutions on condition $b_{\nu 1}^{2}-$ $4 b_{\nu 0} b_{\nu 2} \geq 0$, at that in the case $b_{\nu 0} b_{\nu 2}>0$ there are two solutions ${\Omega^{\prime}}^{2}{ }_{\nu 1,2}$ for $b_{\nu 1} b_{\nu 2}<0$ and no solutions for $b_{\nu 1} b_{\nu 2}>0$, in the case $b_{\nu 0} b_{\nu 2}<0$ there is one solution $\Omega_{\nu 1}^{\prime 2}$. In diamagnets the unstable standing waves exist for the region $q_{\nu}^{2}>q_{\mathrm{c}}^{2}$

\section{Discussion}

In the foregoing the spatial dispersion has been ignored. Calculations show that the spatial dispersion eliminates the instability in high wave number region. Depending on value of the dispersion constant the instability disappears or retains just for an interval of the wave numbers. Amplitudes of the unstable excitations are restricted by nonlinear effects. In a stationary state the number of excitations with opposite momenta and moment of momenta is equal due to conservation laws. When excitation energy dissipates (amplitude decreases) instability arises, as a result energy loss is filled up (amplitude return to initial value). In bounded continuum there are steady states for discrete wave numbers $q_{\nu}^{2}$ located into unstable region.

Above dynamic of the magnetization under fixed temperature is examined. Variable temperature gives additional effects similar to effects in continuum with internal rotation [3]. In particular, thermomagnetic effect and thermomagnetic waves are possible.

As standing waves have different spatial parameters. Therefore in elastically deformed continuum coupled magneto-mechanical oscillations are possible.

Calculations show that taking into account the polarization and current formula for imaginary and real parts frequency and wave number become complicated at that above considered peculiarities in fluctuation spectrum and wave propagation remain.

\section{Acknowledgments}

Author thanks F.V. Lisovskii for fruitful discussions and helpful remarks.

\section{References}

[1] A.G. Gurevich, G.A. Melkov, Magnetization Oscillations and Waves, CRC-Press, London 1996.

[2] A.F. Kabychenkov, Bull. Rus. Acad. Sci. Phys. 74, 1376 (2010)

[3] A.F. Kabychenkov, Techn. Phys. 54, 1116 (2009).

[4] S.R. de Groot, P. Mazur, Non-Equilibrium Thermodynamics, North-Holland, Amsterdam 1962.

[5] I. Gyarmati, Non-Equilibrium Thermodynamics, Springer-Verlag, Berlin 1970.

[6] L.D. Landau, E.M. Lifshits, Electrodynamics of Continuous Media, Pergamon, New York 1992.

[7] S.V. Vonsovskii, Magnetism, Wiley, 1974.

[8] A.F. Kabychenkov, in: Proc. IV Int. Symp. on Surface Waves in Solid and Layered Structures, Eds. Yu.V. Gulyaev, Zh.I. Alferov, St. Petersburg State University of Aerospace Instrumentation, St. Petersburg 1998, p. 83. 